\title{
Associative relatedness effects in retrieval-based and familiarity-based recognition
}

\author{
DAVID L. HORTON and TIMOTHY J. PAVLICK \\ University of Maryland, College Park, Maryland
}

\begin{abstract}
The results of two experiments are reported that involved the encoding-specificity recognitionfailure paradigm. The results of these experiments, using materials that varied in associative relatedness, support the distinction between two qualitatively different recognition processes. Retrieval-based recognition was seen to increase with increases in associative relatedness and was accompanied by successful cued recall. Familiarity-based recognition, however, increased with decreases in associative relatedness and was not accompanied by successful cued recall. It is suggested that these two kinds of recognition reflect different memory systems and that they are often inversely related to one another.
\end{abstract}

Horton, Pavlick, and Moulin-Julian (in press) have proposed a dual-process account of recognition memory that involves a distinction between retrieval-based recognition and familiarity-based recognition. They propose that retrieval-based recognition is an episodic memory process that is similar to the process that influences recall. Familiarity-based recognition, in contrast, operates in terms of sensory-perceptual features in semantic memory, much like those involved in pattern recognition. The notion that recognition is influenced by both retrieval and familiarity is far from new (see Bartlett, 1932; Jacoby \& Dallas, 1981; Mandler, 1980). However, Horton et al. suggest that retrieval-based recognition and familiaritybased recognition not only operate out of different memory systems but that, in terms of performance, they tend to be inversely related to one another and never complement one another.

Two of the experiments reported by Horton et al. involve the use of a paradigm in which target words are tested in both a recognition task and a recall task. In this paradigm, four outcomes are possible. Targets may be remembered (both recognized and recalled), they may be recognized only, recalled only, or forgotten (not recognized or recalled).

The results of these two experiments indicated that performance in the remembered outcome increased as the associative relationship between cue and target members of word pairs presented at study increased. In contrast, performance in the recognized-only outcome increased with decreases in associative relatedness. Performance in the remembered outcome is seen as reflecting retrievalbased recognition, and performance in the recognizedonly outcome reflects familiarity-based recognition. When these two measures are combined to form an index of

Computing services were provided by the Computer Science Center of the University of Maryland. Requests for reprints should be sent to D. L. Horton, Department of Psychology, University of Maryland, College Park, MD 20742. overall recognition performance, no significant relationship to associative relatedness is observed.

The two experiments reported here constitute partial replications of the first two experiments reported by Horton et al. The materials and procedures for the first experiment reported here are identical to those of their first experiment, and those for the second experiment reported here are identical to those of their second experiment. However, in addition to the variation in associative relatedness, Horton et al. also assessed conscious recollection during the recognition task whenever the response "old" was made. This procedure was not employed in either of the experiments reported here.

\section{EXPERIMENT 1}

\section{Method}

Materials. The stimulus materials employed in Experiment 1 were word pairs that were highly asymmetrical in associative strength according to a set of controlled association norms obtained from Douglas L. Nelson (personal communication, November 1983). Twenty-four word pairs, eight at each of three levels of strength, were employed. The highest strength level $(\mathrm{H})$ ranged from .35 to .93 , with an average strength of .57 in the dominant direction and .06 in the opposite direction. An example is alarm-clock, which has an associative strength level of .39 in the alarm-to-clock direction and a .08 strength in the opposite direction. The intermediate strength level $(M)$ involved pairs ranging in strength from .21 to .28 , with an average strength of .24 in the dominant direction and .02 in the opposite direction. An example is rangestove, which has an associative strength level of .21 in the range-tostove direction and a .01 strength in the opposite direction. The lowest strength level $(\mathrm{L})$ involved pairs ranging from .10 to .17 in strength, with an average strength of .14 in the dominant direction and .01 in the opposite direction. An example is bolt-lock, which has an associative strength level of .16 in the bolt-to-lock direction and a .02 strength in the opposite direction. For half of the subjects in this experiment, the dominant strength was from cue to target; for the other half, the dominant strength was from target to cue. Dominant cue-to-target strength was expected to facilitate cued recall but not recognition, whereas dominant target-to-cue strength was expected to facilitate recognition but not cued recall (Mandler, 1980). In further discussion, the direction of dominant strength will be referred to as path direction and the strength level as path strength. 
Subjects. Forty undergraduate students participated in Experiment 1. The students received extra course credit for their participation. Twenty subjects were randomly assigned to each path-direction condition.

Procedure. The subjects were run in small groups. They studied a series of 32 word pairs for $5 \mathrm{sec}$ each. They were instructed to remember the capitalized word (i.e., target) in each pair and to look for a relationship between the words in each pair in order to help them remember the target word. The first and last four word pairs constituted a primacy-recency buffer, and these words did not appear again in the experiment. A 10-min numerical problem-solving distractor task immediately followed presentation of the pairs.

Following the problem-solving task, the subjects were asked to complete the recognition task, which consisted of the 24 target words and 24 distractor words. They were instructed to identify each word as old (i.e., target) or new (i.e., a distractor). The distractors were identical for both groups of subjects. After the recognition task was completed, the subjects performed a cued-recall task. The context word for each of the 24 critical pairs presented during encoding served as the retrieval cue, and the subjects were asked to write down a target word in the space provided next to each context word.

\section{Results and Discussion}

The data from this experiment were analyzed in terms of the number of target words at each strength level in the outcomes designated as remembered, recognized only, and forgotten. The results for recalled only will be reported in a different, and more appropriate, context. For clarity of presentation, the results are reported as proportions. The findings for Experiment 1 are shown in Table 1.

The results of interest are for the remembered and recognized-only outcomes. The results for the forgotten outcome are presented to indicate that forgetting generally increases as path strength declines.

As can be seen in Table 1, performance in the remembered outcome is inversely related to performance in the recognized-only outcome. If overall recognition performance was at or near ceiling, it could be argued that the inverse relationship was an artifact due to item selection. Clearly, for a fixed number of correctly recognized target words, the more that occur in the remembered outcome, the fewer there can be in the recognized-only outcome. However, overall recognition was not at or near ceiling. A more complete explication of this issue can be found in Horton et al. (in press).

Table 1

Proportion of Targets in Each Memory Outcome as a Function of Path Direction and Path Strength

\begin{tabular}{cccc}
\hline & \multicolumn{3}{c}{ Memory Outcome } \\
Strength & Remembered & $\begin{array}{c}\text { Recognized } \\
\text { Only }\end{array}$ & Forgotten \\
\cline { 2 - 4 } & \multicolumn{4}{c}{ Path Direction: Cue to Target } \\
H & .76 & .02 & .01 \\
M & .58 & .11 & .13 \\
L & .59 & .13 & .09 \\
& Path Direction: Target to Cue \\
H & .68 & .20 & .10 \\
M & .59 & .28 & .10 \\
L & .38 & .46 & .13
\end{tabular}

Note- $\mathrm{H}=$ highest strength level; $\mathbf{M}=$ intermediate strength level; $\mathrm{L}=$ lowest strength level.
It can be seen in Table 1 that many more target items were remembered than were recognized only. It can also be seen that remembered performance generally increased with path strength, but the increase was more apparent when path direction goes from target to cue. In the recognized-only outcome, performance clearly increased as path strength declined in both path-direction conditions. In the recognized-only outcome, performance was better in the target-to-cue path-direction condition, whereas the opposite was the case for the remembered outcome. These observations are supported by an analysis of variance (ANOVA) that indicated significant effects for path direction $[F(1,38)=12.42, p<.01]$, memory outcome $[F(1,38)=108.47, p<.0001]$, memory outcome $\times$ path strength $[F(2,76)=32.39, p<.0001]$, and path direction $\times$ memory outcome $\times$ path strength $[F(2,76)$ $=7.13, p<.01]$. It should be noted that there was no main effect of path strength, nor did path strength interact with path direction. No significant effects were observed in the forgotten outcome.

These results clearly replicate those reported by Horton et al. in their Experiment 1. The findings also support the distinction between retrieval-based and familiarity-based recognition as qualitatively different processes that tend to be inversely related.

One final bit of statistical information concerns the comparability of the subjects in the two path-direction conditions. The recognition distractor items were the same for both groups of subjects, and a comparison of performance in terms of correct rejections indicated that both groups performed the same (.89). This observation is important because it suggests that the between-group effects noted previously really were due to path direction and not to differences between the two groups of subjects.

\section{EXPERIMENT 2}

\section{Method}

The only methodological difference between Experiment 2 and Experiment 1 was that, in Experiment 2, the Diehl and Horton (1988) related distractor task was employed in recognition. For each target word, there were three distractor words associatively linked to both the cue and the target member of each pair. It was expected that the related distractor task would lower overall performance, relative to that in Experiment 1 , in both the recognition task and the following cued-recall task. It was anticipated that this would lower the frequency of the remembered outcome and raise the frequency of the forgotten outcome. While performance in the remembered outcome was expected to be positively related to path strength, as was the case in Experiment 1, performance in the forgotten outcome was expected to be inversely related to path strength. In Experiment 1, performance in the forgotten outcome was uniformly low, and no significant relationship to path strength was observed. In addition, the frequency of the recognized-only outcome was also expected to increase with path direction going from cue to target. In Experiment 2, there were 22 subjects in each path-direction condition.

\section{Results and Discussion}

The data for Experiment 2 are reported and analyzed in the same manner as in Experiment 1. The results of Experiment 2 for the remembered, recognized-only, and forgotten outcomes are shown in Table 2. 
Table 2

Proportion of Targets in Each Memory Outcome as a Function of Path Direction and Path Strength

\begin{tabular}{cccc}
\hline & \multicolumn{3}{c}{ Memory Outcome } \\
\cline { 2 - 4 } $\begin{array}{c}\text { Path } \\
\text { Strength }\end{array}$ & Remembered & $\begin{array}{c}\text { Recognized } \\
\text { Only }\end{array}$ & Forgotten \\
\hline & Path Direction: Cue to Target \\
H & .60 & .10 & .06 \\
M & .43 & .14 & .15 \\
L & .45 & .18 & .22 \\
& Path Direction: Target to Cue & \\
H & .57 & .19 & .17 \\
M & .47 & .30 & .21 \\
L & .30 & .38 & .27 \\
\hline
\end{tabular}

Note $-\mathrm{H}=$ highest strength level; $\mathrm{M}=$ intermediate strength level; $\mathrm{L}=$ lowest strength level.

It is apparent from Table 2 that the main findings obtained in Experiment 1 are replicated. As can be seen in Table 2, more target items were remembered than were recognized only. Again, performance in the remembered outcome generally increased with increases in path strength, and the increase was more clearly apparent when path direction went from target to cue than when it went from cue to target. In the recognized-only outcome, however, performance increased as path strength declined in both path-direction conditions. In the recognized-only outcome, performance was better in the target-to-cue path direction than in the cue-to-target path direction. As expected, due to differential increases in the recognized-only and forgotten outcomes in the cue-to-target path-direction condition, performance in the remembered outcome showed no effect of path direction. These observations are supported by an ANOVA that indicated significant effects for path direction $[F(1,42)=5.39, p<.03]$, memory outcome $[F(1,42)=36.56, p<.0001]$, and memory outcome $\times$ path strength $[F(2,84)=18.11$, $p<.0001]$. As in Experiment 1, the inverse relationship between performance in the remembered and recognizedonly outcomes across levels of path strength resulted in no main effect of path strength. There was no interaction between path strength and path direction.

As in Experiment 1, the remembered outcome occurred more often as path strength increased, and this result was more clearly shown when path direction was from target to cue. When path direction was from cue to target, the facilitation of cued recall was sufficient to override the effects of path strength between the low and intermediate conditions. As previously noted, the override was also attributable to the fact that the path-strength difference between the intermediate (.24) and low (.14) conditions was much smaller than that between the intermediate and high (.57) conditions.

An expected but important finding in Experiment 2 concerns the observation that the frequency of the forgotten outcome increased as path strength declined in both pathdirection conditions. It should also be noted that the for- gotten outcome was greater for the target-to-cue path direction. Both of these observations are in line with performance in the recognized-only outcome, and they are supported by an ANOVA that revealed significant effects for path direction $[F(1,42)=4.66, p<.04]$ and path strength $[F(2,84)=11.65, p<.0001]$.

The results of Experiment 2 go a long way in supporting our interpretation that there are two kinds of recognition memory that are based on different processes and, furthermore, that improvements in performance in these two kinds of recognition memory are often inversely related to one another. As we saw in Experiment 1, retrievalbased recognition improves with increases in cued recall and with increasing quality of episodic information. However, familiarity-based recognition shows improvements in performance that are not accompanied by successful cued recall but do accompany declines in the quality of episodic information. In Experiment 2, this interpretation is most strongly supported by the observation that increases in the recognized-only outcome and increases in the forgotten outcome both accompany decreases in path strength in both path-direction conditions. It is perfectly reasonable to suggest the forgotten outcome should occur more often as path strength and the quality of episodic information decline. However, that also requires the conclusion that the increases in the recognized-only outcome that accompany declines in path strength also accompany declines in the quality of episodic information. We will have more to say about the meaning of this interpretation in the general discussion.

One other difference between the results of Experiments 1 and 2 should be noted, even though statistical comparisons were not carried out. In Experiment 2, the recognizedonly outcome in the cue-to-target path-direction condition occurred more often than it did in Experiment 1. We interpret this difference as being due to lower quality episodic information available in Experiment 2 (due to the related distractor recognition task), which led to an increase in familiarity-based recognition hits in this experiment. When path direction goes from target to cue, no difference between Experiments 1 and 2 is found for the recognizedonly outcome. This result most likely occurs because the increased level of the forgotten outcome in Experiment 2 puts the recognized-only outcome at a ceiling.

\section{GENERAL DISCUSSION}

The results of the experiments reported here clearly support the distinction between retrieval-based and familiarity-based recognition as qualitatively different processes that tend to be inversely related in terms of memory performance. In both experiments, performance in the remembered outcome increased with increases in path strength, as would be expected since path strength increases should result in a higher quality of episodic information. In contrast, performance in the recognizedonly outcome decreased as path strength increased. The same pattern was found for the forgotten outcome in Experiment 2, suggesting that recognition-only performance is best with low-quality episodic information. These results clearly replicate the findings reported by Horton et al. (in press) for the path-strength and path-direction variables across memory outcomes. 


\section{REFERENCES}

Bartlett, F. C. (1932). Remembering. Cambridge: Cambridge University Press.

Diehl, V. A., \& Horton, D. L. (1988). Encoding context effects in recognition and cued recall. Bulletin of the Psychonomic Society, 26, 393-394.

Horton, D. L., Pavlick, T. J., \& Moulin-Julian, M. W. (in press). Retrieval-based and familiarity-based recognition and the quality of information in episodic memory. Journal of Memory \& Language.
JACOBY, L. L., \& DALLAS, M. (1981). On the relationship between autobiographical memory and perceptual learning. Journal of Experimental Psychology: General, 110, 306-340.

MANDLER, G. (1980). Recognizing: The judgment of previous occurrences. Psychological Review, 87, 252-271.

(Manuscript received July 18, 1992.) 